\title{
Multiagent Decision by Partial Evaluation
}

\author{
Yang Xiang and Frank Hanshar \\ University of Guelph, Canada
}

\begin{abstract}
We consider multiagent cooperative decision in stochastic environments, and focus on online decision during which agents communicate. We generalize partial evaluation from a specific application to a class of collaborative decision networks (CDNs), and propose a distributed decision algorithm based on partial evaluation. We show that when agents have private decision variables, the new algorithm can significantly speed up decision in comparison with the earlier CDN algorithm.
\end{abstract}

\section{Introduction}

We consider a class of multiagent cooperative decision problems in partially observable and stochastic environments. One example is collaborative design in supply chain [5]. Another example is multiagent expedition (MAE) [7]. We assume that the decision problems can be modeled as CDNs [6,7], a class of decision-theoretic, cooperative, multiagent, graphical models. Main assumptions of CDNs are the following: Application environment (env, a set of variables) is decomposed into overlapping sub-environments (subenvs), that can be organized into a hypertree with running intersection property. Each subenv is hosted by an agent $A$ and consists of decision variables, effect variables, and utility variables. Dependency among variables in each subenv is modeled as a decision subnet (influence diagram). Overlaps of subenvs (agent interfaces) consist of decision variables only.

We focus on online decision making over short horizons rather than offline policy making, e.g., $[4,2]$. That is, agents decide the best joint action based on what they know about the current env. Focus on current env state, rather than all possible trajectories as in offline policy making, allows much more efficient decision making.

An agent makes a simultaneous decision [10] when it decides its actions over multiple decision variables, all of which have the horizon length $h=1$. A collection of decisions one from each agent is a joint decision. This work concerns joint, simultaneous decisions. An example is collaborative design in supply chain, where the component design at each agent is a simultaneous decision and the product design is a joint, simultaneous decision. This work concerns also sequential decision, where agents decide and act over time, e.g., in MAE. Agents plan at every time step with $h \geq 1$ and act according to joint action for time $t=1$.

We term each choice of a decision variable as an action, e.g., choosing $2 G B$ RAM for a device under design. For multiple decision variables (possibly for different time), we refer to a vector of choices, one for each variable, as a plan. If these variables are contained in a single subenv, the plan is local, otherwise, it is joint. Hence, a component design in collaborative design is a local plan, and so is a 
sequence of $h>1$ movements by a MAE agent. A product design is a joint plan, and so is a collection of local plans of a team of MAE agents. The decision task is to obtain an optimal joint plan by distributed online computation.

The above decision problem is equivalent to a Dec-POMDP [1,8], and hence its optimal solution is generally intractable. During online decision, our agents communicate, as in e.g. [3], but not local observations. For our decision algorithm, we analyze its communication cost, while our agents do not during decision making. To improve efficiency in MAE applications, an idea of partial evaluation was explored in [9]. Significant speedup was achieved in centralized decision, while application to distributed decision was not productive. This work generalizes partial evaluation to a class of CDNs, where each directed path starts from a decision node, followed by an effect node, and ends by a utility node. We extend the earlier algorithm for CDNs [6] based on partial evaluation, and show that when subenvs contain private decisions the new algorithm can significantly speed up decision making.

Section 2 introduces CDNs. Sections 3 through 5 present partial evaluation in subnets of increasing sophistication. The proposed algorithm is presented in Sections 6 through 8, with performance demonstrated in Section 9.

\section{Background}

A set $\mathscr{A}$ of $n$ agents populates an env. It is represented as a set $\mathscr{V}$ of variables, decomposed into overlapping subenvs $V_{1}, \ldots, V_{n}$, each hosted by an agent. The decomposition allows the construction of a hypertree $\mathscr{H}$ whose (hyper)nodes are labeled by subenvs such that intersection of every two nodes is contained in each node on the path between the two (running intersection).

Each subenv $V=D \cup E \cup U$, where $D, E, U$ are disjoint, is modeled by an agent $A \in \mathscr{A}$ into a decision subnet $S=(D, E, U, G, P, T)$, where $G$ is an acyclic directed graph whose nodes are labeled by elements of $V$. D is a set of local decision variables $D=\left\{d_{1}, d_{2}, \ldots\right\}$, and we denote $\rho=|D|$. Each $d_{i}$ has a finite space of options or actions $O p_{i}=\left\{d_{i 1}, d_{i 2}, \ldots\right\}$. Denote $\sigma=\max _{i}\left|O p_{i}\right|$.

$E$ is a set of effect variables $E=\left\{e_{1}, e_{2}, \ldots\right\}$, representing outcomes of actions. Each $e_{i}$ has a finite space $E f_{i}=\left\{e_{i 1}, e_{i 2}, \ldots\right\}$. Denote $\kappa=\max _{j}\left|E f_{j}\right|$. Each $e_{i}$ has a set $\delta_{i} \subseteq D$ of decision variables as its parents in $G$. The dependency of $e_{i}$ on $\delta_{i}$ is quantified by a conditional probability table (CPT) $P\left(e_{i} \mid \delta_{i}\right) . P$ is the set of CPTs, one for each $e_{i} \in E$.

$U$ is a set of utility variables $U=\left\{u_{1}, u_{2}, \ldots\right\}$, encoding the subjective preference of $A$ over effects, and we denote $\eta=|U|$. Each $u_{i} \in U$ has a set $\pi_{i} \subseteq E$ of effects as parents in $G$. Denote $m=\max _{i}\left|\pi_{i}\right|$. Preference over $\pi_{i}$ is encoded in a utility function $u_{i}\left(\pi_{i}\right) \in[0,1]$, and $T$ is a set of functions, one for each $u_{i} \in U$. Each $u_{i}$ is associated with a weight $w_{i}>0$ such that $\sum_{i} w_{i}=1$.

If subenv $V$ hosted by agent $A$ is adjacent on the hypertree to subenv $V^{\prime}$ hosted by $A^{\prime}$, then the (hyper)link labeled $V \cap V^{\prime}$ is the interface of $A$ and $A^{\prime}$. For $A$, the interface is public, and all other variables in $V$ are private relative to $A^{\prime}$.

A $\mathrm{CDN}$ is a tuple $(\mathscr{A}, \mathscr{V}, \mathscr{H}, \mathscr{S})$, where $\mathscr{S}$ is a set of decision subnets, one for each $A \in \mathscr{A}$. An example is in Fig. 1, where nodes in $D, E, U$ are drawn as squares, ovals, diamonds, respectively. Defined as above, every directed path in a subnet has a length 2, e.g., Fig. 1 (a). This work focuses on such CDNs. More general subnets can all be converted equivalently to length-2 (with modification to relevant CPTs and utility functions), and we assume that such is done. 


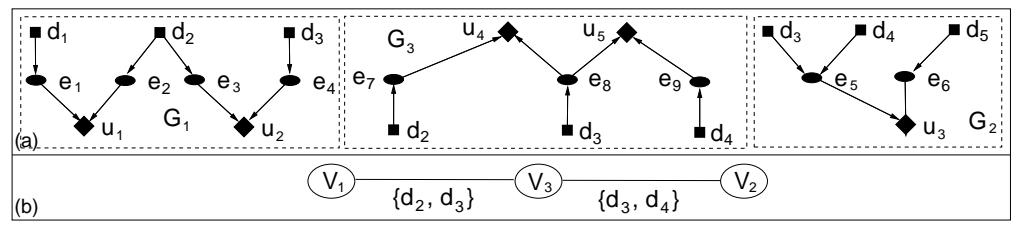

Fig. 1. (a) CDN subnets. (b) Hypertree.

\section{Single Decision Variable}

Consider a decision subnet where $\rho=1, \eta=1$, and $V=\left\{d_{i}, e_{i}, u_{i}\right\}$. Expected utility of taking an action $d_{i}=d_{i j}$ is

$$
e u\left(d_{i j}\right)=P\left(e_{i 1} \mid d_{i j}\right) u_{i}\left(e_{i 1}\right)+P\left(e_{i 2} \mid d_{i j}\right) u_{i}\left(e_{i 2}\right)+\ldots,
$$

which requires $\kappa$ probability retrievals, $\kappa$ utility retrievals, $\kappa$ multiplications, and $\kappa-1$ additions. Action $d_{i j}$ is fully evaluated when $e u\left(d_{i j}\right)$ is computed. Its complexity is $O(\kappa)$. Agent's objective is to compute the optimal decision pair $\left(d_{i k}^{*}\right.$, meuv $)$ such that $e u\left(d_{i k}^{*}\right)=$ meuv $=\max _{j} e u\left(d_{i j}\right)$. This generally involves a full evaluation of each $d_{i j}$, with the complexity $O(\sigma \kappa)$.

For every action, we refer to an effect with the highest probability (indexed once and referenced repeatedly) as the pivot effect, and break ties arbitrarily. Let $e_{i k}$ be the pivot effect of action $d_{i k}$. Then $e u\left(d_{i k}\right) \leq P\left(e_{i k} \mid d_{i k}\right) u_{i}\left(e_{i k}\right)+(1-$ $\left.P\left(e_{i k} \mid d_{i k}\right)\right) u_{i}^{\max } \equiv Q_{i k}$, where $u_{i}^{\max }=\max _{x} u_{i}\left(e_{i x}\right)$. We say that $d_{i j}$ dominates $d_{i k}$ iff $e u\left(d_{i j}\right)>e u\left(d_{i k}\right)$. If $Q_{i k}<e u\left(d_{i j}\right)$, then $e u\left(d_{i k}\right)<e u\left(d_{i j}\right)$ and $d_{i k}$ is dominated by $d_{i j}$. Computing $Q_{i k}$ requires one probability retrieval, one utility retrieval, two multiplications, and two additions. Action $d_{i k}$ is partially evaluated when $Q_{i k}$ is computed.

Suppose probabilities of pivot effects for $d_{i}$ are nearly identical. In MAE, a movement decision has alternatives north, east, etc. Moving north most likely lands on north location. Its probability is about the same as that of landing on east location if moving east. In many envs, each action most likely produces a given effect, with smaller probabilities to produce other effects. As another example, a message may be sent by email or post. Emails most likely arrive in seconds while post mails most likely in days. Formally, we assume

$$
\forall j, k P\left(e_{i j} \mid d_{i j}\right)=P\left(e_{i k} \mid d_{i k}\right)=p .
$$

Partial evaluation of action $d_{i k}$ amounts to compute $p u_{i}\left(e_{i k}\right)+(1-p) u_{i}^{\max }$. To determine whether

$$
p u_{i}\left(e_{i k}\right)+(1-p) u_{i}^{\max }<e u\left(d_{i j}\right)
$$

holds, we check instead whether $u_{i}\left(e_{i k}\right)<\frac{e u\left(d_{i j}\right)}{p}-\frac{1-p}{p} u_{i}^{\max }$ holds. We assume that the right hand side (threshold) has been obtained before $d_{i k}$ is evaluated. Then if the above inequality holds, $d_{i k}$ can be rejected with just one utility retrieval and one comparison, and the threshold can be reused for evaluating the next action. In this case, partial evaluation of $d_{i k}$ has a complexity of $O(1)$.

This leads to the following partial evaluation based decision to obtain $\left(d_{i k}^{*}\right.$, meuv $)$ : Apply full evaluation for the first action to establish a threshold. For each alternative action, apply partial evaluation to reject if warranted. Otherwise, apply full evaluation to it and update the threshold. The last option accepted is $d_{i k}^{*}$.

Using this method, efficiency is gained by evaluating a $d_{i k}$ fully only if the above inequality fails. Let $\theta \in[0,1]$ be the percentage of $d_{i k}$ fully evaluated. Then the complexity of partial evaluation based decision is $O(\theta \sigma \kappa+(1-\theta) \sigma)$. 


\section{Multiple Decision Variables}

Consider the case where $\rho>1$ and $\eta=1$, e.g., subnet $G_{2}$ in Fig. 1 (a), where each local plan is to be evaluated. Let $\bar{d}$ be a local plan over $D, \bar{e}$ be its (compound) pivot effect (made of pivot effect of each action in $\bar{d}$ ), $\bar{e}^{\prime \prime}$ be any (compound) effect, and $e u(\bar{d})$ be the expected utility of $\bar{d}$. Then

$$
e u(\bar{d})=P(\bar{e} \mid \bar{d}) u_{i}(\bar{e})+\sum_{\overline{e^{\prime \prime}}} P\left(\overline{e^{\prime \prime}} \mid \bar{d}\right) u_{i}\left(\overline{e^{\prime \prime}}\right) .
$$

Let $\beta$ be a subset of $D$ and $\gamma=D \backslash \beta$. We consider the decision problem to obtain a pair of functions $(\operatorname{meu}(\beta)$, peer $(\beta))$, where meu $: \beta \rightarrow[0,1]$ and peer $: \beta \rightarrow \gamma$, such that for each plan $\bar{b}$ over $\beta, \operatorname{meu}(\bar{b})=\max _{\bar{y}} e u(\bar{b}, \bar{y})$ and eu $(\bar{b}, \operatorname{peer}(\bar{b}))=$ $m e u(\bar{b})$, where $\bar{y}$ is a plan over $\gamma,(\bar{b}, \bar{y})$ denotes a join of plans, maximization is over each plan $\bar{y}$, and peer $(\bar{b})$ equals the optimal plan $\bar{y}^{*}$. We refer to $\beta$ as constraint scope and $\gamma$ as optimization scope. In other words, $($ meu $(\beta)$, peer $(\beta))$ specifies, for each constraint $\bar{b}$, the MEU meu $(\bar{b})$ and the corresponding optimal plan $\overline{d^{*}}=\left(\bar{b}, \operatorname{peer}(\bar{b})=\overline{y^{*}}\right)$.

The task requires evaluating $e u(\bar{b}, \bar{y})$. Denote plan projection by $\bar{b}=\operatorname{proj}(\bar{d}, \beta)$, $\bar{y}=\operatorname{proj}(\bar{d}, \gamma)$, and write $\bar{d}=(\bar{b}, \bar{y})$. To evaluate $e u(\bar{d})$ by Eqn. (3), $P\left(\overline{e^{\prime \prime}} \mid \bar{d}\right)$ must be computed for each $\overline{e^{\prime \prime}}$. For $G_{2}$ in Fig. 1 (a), $P\left(\overline{e^{\prime \prime}} \mid \bar{d}\right)=P\left(e_{5}, e_{6} \mid d_{3}, d_{4}, d_{5}\right)=$ $P\left(e_{5} \mid d_{3}, d_{4}\right) P\left(e_{6} \mid d_{5}\right)$. In general, computation of $P\left(\overline{e^{\prime \prime}} \mid \bar{d}\right)$ for each $\overline{e^{\prime \prime}}$ involves $m$ probability retrievals and $m-1$ multiplications. Hence, a full evaluation of $\bar{d}$ by Eqn. (3) takes $m \kappa^{m}$ probability retrievals, $\kappa^{m}$ utility retrievals, $m \kappa^{m}$ multiplications, and $\kappa^{m}-1$ additions. Complexity of full evaluation of $\bar{d}$ is thus $O\left(m \kappa^{m}\right)$. To obtain meu $(\beta)$ by full evaluation, a total of $\sigma^{\rho}$ alternative $\bar{d}$ must be evaluated, and the complexity is thus $O\left(\sigma^{\rho} m \kappa^{m}\right)$.

For more efficient decision, we observe

$$
\begin{aligned}
e u(\bar{d}) & =P(\bar{e} \mid \bar{d}) u_{i}(\bar{e})+\sum_{\overline{e^{\prime \prime}}} P\left(\overline{e^{\prime \prime}} \mid \bar{d}\right) u_{i}\left(\overline{e^{\prime \prime}}\right) \leq P(\bar{e} \mid \bar{d}) u_{i}(\bar{e})+\sum_{\overline{e^{\prime \prime}}} P\left(\overline{e^{\prime \prime}} \mid \bar{d}\right) u_{i}^{\max }(4) \\
& =P(\bar{e} \mid \bar{d}) u_{i}(\bar{e})+(1-P(\bar{e} \mid \bar{d})) u_{i}^{\max }, \text { where } u_{i}^{\max }=\max _{\overline{e^{\prime \prime}}} u_{i}\left(\overline{e^{\prime \prime}}\right)
\end{aligned}
$$

Let $e_{i}$ be an effect variable with parent set $\delta_{i}$. Let $\overline{\delta_{i k}}$ be $k$ th configuration of $\delta_{i}$ and $e_{i k}$ be its pivot effect. Assume

$$
\forall i, j, k P\left(e_{i j} \mid \overline{\delta_{i j}}\right)=P\left(e_{i k} \mid \overline{\delta_{i k}}\right) .
$$

That is, for each effect variable and its influencing decision variables, probabilities of pivot effects are approximately identical. It then follows that $\forall \bar{d}, \bar{d}^{\prime} P(\bar{e} \mid \bar{d})=$ $P\left(\overline{e^{\prime}} \mid \overline{d^{\prime}}\right)=p$, where $\overline{e^{\prime}}$ is the pivot effect of local plan $\overline{d^{\prime}}$, and $p$ can be computed once for all pivot effects. For the example on $G_{2}$ in Fig. 1, suppose probabilities of pivot effects for $P\left(e_{5} \mid d_{3}, d_{4}\right)$ is 0.7 and those for $P\left(e_{6} \mid d_{5}\right)$ is 0.9 . Then we have $p=0.63$. Now, inequation (4) becomes $e u(\bar{d}) \leq p u_{i}(\bar{e})+(1-p) u_{i}^{\max }$.

In general, given $e u\left(\overline{d^{\prime}}\right)$ and an alternative plan $\bar{d}$, if

$$
p u_{i}(\bar{e})+(1-p) u_{i}^{\max }<e u\left(\overline{d^{\prime}}\right)
$$

it follows that $e u(\bar{d})<e u\left(\overline{d^{\prime}}\right)$ and $\bar{d}$ is dominated by $\overline{d^{\prime}}$. Partial evaluation of $\bar{d}$ by Eqn. (6) takes only one utility retrieval, with complexity $O(1)$.

Extending partial evaluation based decision in Section 3 with the above, the decision problem to obtain $(\operatorname{meu}(\beta)$, peer $(\beta))$ can be solved by PEUtilDec1: 


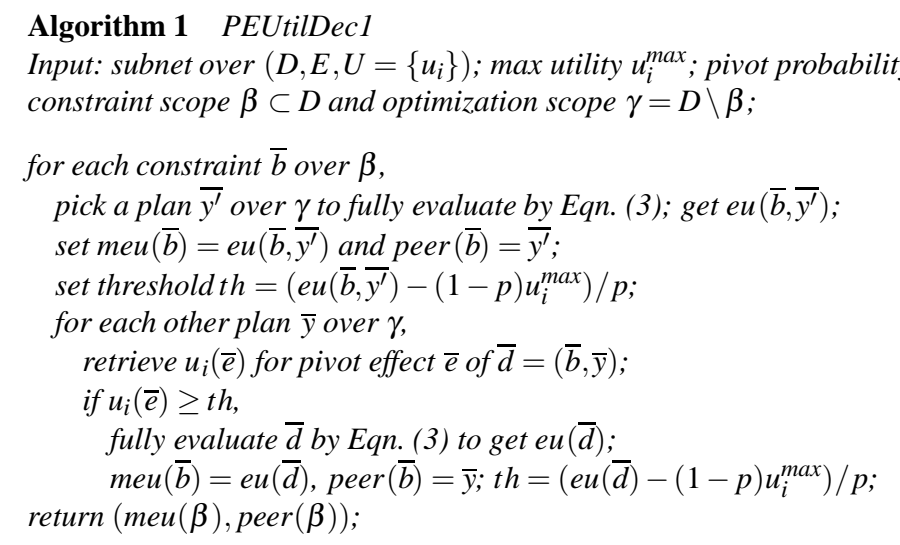

When $\beta=\emptyset$, in the return value of PEUtilDec $1, \operatorname{meu}(\beta)$ becomes a single value and $\operatorname{peer}(\beta)$ becomes a single optimal plan $\overline{d^{*}}$ over $D$. Let $\theta \in[0,1]$ be the percentage of $\bar{d}$ fully evaluated by PEUtilDec1. Its complexity is then $O\left(\theta \sigma^{\rho} m \kappa^{m}+\right.$ $\left.(1-\theta) \sigma^{\rho}\right)=O\left(\theta \sigma^{\rho} m \kappa^{m}\right)$. Proposition 1 summarizes key properties of PEUtilDec1.

Proposition 1 PEUtilDecl satisfies the following: (1) For each constraint $\bar{b}$ over $\beta$, meu $(\bar{b})$ is the MEU. (2) For each $\bar{b}$, peer $(\bar{b})$ is the optimal plan over $\gamma$. (3) Its complexity is $O\left(\theta \sigma^{\rho} m \kappa^{m}\right)$.

\section{Multiple Utility Variables}

Next, consider a decision subnet where $\rho>1$ and $\eta>1$. An example is $G_{1}$ in Fig. 1 (a), where $\eta=2$ and a weight $w_{i}$ is associated with each $u_{i}(i=1,2)$. The optimal local plan cannot be obtained by solving independent sub-problems over $\left\{d_{1}, d_{2}\right\}$ and over $\left\{d_{2}, d_{3}\right\}$. Hence, fully evaluating a local plan amounts to compute

$$
\begin{aligned}
& e u\left(d_{1}, d_{2}, d_{3}\right)= \\
& \quad w_{1} \sum_{e_{1}, e_{2}} P\left(e_{1} \mid d_{1}\right) P\left(e_{2} \mid d_{2}\right) u_{1}\left(e_{1}, e_{2}\right)+w_{2} \sum_{e_{3}, e_{4}} P\left(e_{3} \mid d_{2}\right) P\left(e_{4} \mid d_{3}\right) u_{2}\left(e_{3}, e_{4}\right) .
\end{aligned}
$$

In general, let $\bar{d}$ be a local plan over $D, \bar{e}$ be its pivot effect, $\overline{e^{\prime \prime}}$ be any alternative effect, and $e u(\bar{d})$ be expected utility of $\bar{d}$. For each $u_{i}(i=1, \ldots, \eta)$ with parents $\pi_{i}$, let $\alpha_{i}$ be the set of decision ancestors of $u_{i}$. In $G_{1}$ of Fig. 1 (a), $\alpha_{1}$ for $u_{1}$ is $\left\{d_{1}, d_{2}\right\}$. Define $\overline{e_{i}}=\operatorname{proj}\left(\bar{e}, \pi_{i}\right)$, and $\overline{d_{i}}=\operatorname{proj}\left(\bar{d}, \alpha_{i}\right)$. Then, a full evaluation of $\bar{d}$ computes

$$
e u(\bar{d})=\sum_{i=1}^{\eta} w_{i}\left[P\left(\overline{e_{i}} \mid \overline{d_{i}}\right) u_{i}\left(\overline{e_{i}}\right)+\sum_{\overline{e_{i}^{\prime \prime}}} P\left(\overline{e_{i}^{\prime \prime}} \mid \overline{d_{i}}\right) u_{i}\left(\overline{e_{i}^{\prime \prime}}\right)\right] .
$$

We consider the decision problem to obtain $(\operatorname{meu}(\beta)$, peer $(\beta))$ such that for each plan $\bar{b}$ over $\beta, \operatorname{meu}(\bar{b})=\max _{\bar{y}} e u(\bar{b}, \bar{y})$ and $e u(\bar{b}, \operatorname{peer}(\bar{b}))=\operatorname{meu}(\bar{b})$, where $\bar{y}$ is a plan over $\gamma$, and peer $(\bar{b})$ equals optimal plan $\overline{y^{*}}$. 
Extending result from Section 4, the complexity to fully evaluate $\bar{d}$ is $O\left(\eta m \kappa^{m}\right)$. We consider partial evaluation with the above example: If $e_{j}$ is the child node of $d_{i}$, we denote its pivot effect corresponding to $d_{i k}$ by $e_{j k}$. We have

$$
\begin{aligned}
& e u\left(d_{1 x}, d_{2 y}, d_{3 z}\right) \leq \\
& \quad w_{1} P\left(e_{1 x} \mid d_{1 x}\right) P\left(e_{2 y} \mid d_{2 y}\right) u_{1}\left(e_{1 x}, e_{2 y}\right)+w_{2} P\left(e_{3 y} \mid d_{2 y}\right) P\left(e_{4 z} \mid d_{3 z}\right) u_{2}\left(e_{3 y}, e_{4 z}\right) \\
& \quad+w_{1}\left(1-P\left(e_{1 x} \mid d_{1 x}\right) P\left(e_{2 y} \mid d_{2 y}\right)\right) u_{1}^{\max }+w_{2}\left(1-P\left(e_{3 y} \mid d_{2 y}\right) P\left(e_{4 z} \mid d_{3 z}\right)\right) u_{2}^{\max } .
\end{aligned}
$$

Assuming probabilities of pivot effects for each effect variable $e_{i}$ is identical, denoted by $p_{i}$, we have $e u\left(d_{1 x}, d_{2 y}, d_{3 z}\right) \leq w_{1} p_{1} p_{2} u_{1}\left(e_{1 x}, e_{2 y}\right)+w_{2} p_{3} p_{4} u_{2}\left(e_{3 y}, e_{4 z}\right)+$ $w_{1}\left(1-p_{1} p_{2}\right) u_{1}^{\max }+w_{2}\left(1-p_{3} p_{4}\right) u_{2}^{\max }$.

Given $e u\left(d_{1 x}^{\prime}, d_{2 y}^{\prime}, d_{3 z}^{\prime}\right)$ and another plan $\left(d_{1 x}, d_{2 y}, d_{3 z}\right)$, if

$$
\begin{gathered}
w_{1} p_{1} p_{2} u_{1}\left(e_{1 x}, e_{2 y}\right)+w_{2} p_{3} p_{4} u_{2}\left(e_{3 y}, e_{4 z}\right)+w_{1}\left(1-p_{1} p_{2}\right) u_{1}^{\max } \\
+w_{2}\left(1-p_{3} p_{4}\right) u_{2}^{\max }<e u\left(d_{1 x}^{\prime}, d_{2 y}^{\prime}, d_{3 z}^{\prime}\right),
\end{gathered}
$$

holds, it follows that $e u\left(d_{1 x}, d_{2 y}, d_{3 z}\right)<e u\left(d_{1 x}^{\prime}, d_{2 y}^{\prime}, d_{3 z}^{\prime}\right)$, and $\left(d_{1 x}, d_{2 y}, d_{3 z}\right)$ is dominated by $\left(d_{1 x}^{\prime}, d_{2 y}^{\prime}, d_{3 z}^{\prime}\right)$. Partial evaluation of $\left(d_{1 x}, d_{2 y}, d_{3 z}\right)$ by Eqn. (8) takes only two utility retrievals.

In general, each utility variable $u_{i}$ has $\pi_{i}$ effect parents, each of which is associated with a pivot probability $p_{i j}$ indexed by $j$. Given $e u\left(\overline{d^{\prime}}\right)$ from a full evaluation, and an alternative plan $\bar{d}$, if

$$
\sum_{i=1}^{\eta} w_{i}\left(\left(\prod_{j} p_{i j}\right) u_{i}\left(\overline{e_{i}}\right)+\left(1-\left(\prod_{j} p_{i j}\right)\right) u_{i}^{\max }\right)<e u\left(\overline{d^{\prime}}\right),
$$

then $\bar{d}$ is dominated by $\overline{d^{\prime}}$. This leads to threshold and dominance comparison below:

$$
\begin{gathered}
t h=e u\left(\overline{d^{\prime}}\right)-\sum_{i=1}^{\eta} w_{i}\left(1-\left(\prod_{j} p_{i j}\right)\right) u_{i}^{\max } \\
\sum_{i=1}^{\eta} w_{i}\left(\prod_{j} p_{i j}\right) u_{i}\left(\overline{e_{i}}\right)<t h .
\end{gathered}
$$

By pre-computing $w_{i}\left(\prod_{j} p_{i j}\right)$ for each $i$, partial evaluation of $\bar{d}$ has a complexity of $O(\eta)$. Extending PEUtilDec1, PEUtilDec2 makes partial evaluation based decision with multiple decision and utility variables, whose properties are stated below.

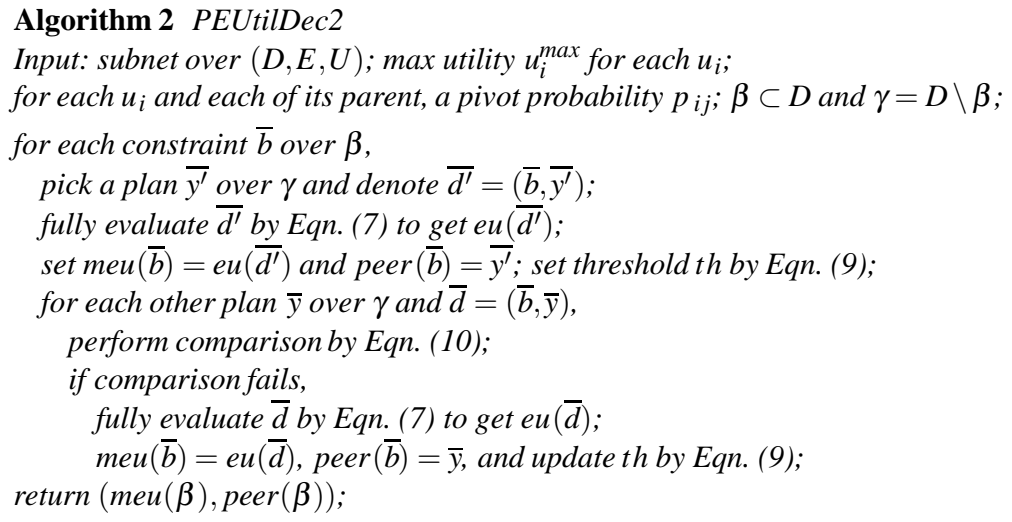


Proposition 2 PEUtilDec2 satisfies (1) and (2) of Proposition 1, as well as the following: Its complexity is $O\left(\theta \sigma^{\rho} \eta m \kappa^{m}\right)$.

Note that, when $\beta=\emptyset$, the return value of $\operatorname{PEUtilDec} 2, \operatorname{meu}(\beta)$, becomes a single value, and $\operatorname{peer}(\beta)$ becomes a single optimal plan $\overline{d^{*}}$ over $D$.

\section{Utility Message by Leaf Agent}

We obtain an optimal joint plan with two rounds of message passing, using PEUtilDec1 and PEUtilDec2. Hypertree is directed from an arbitrary root and agents become parent-child according to the direction. In first round, utility messages flow from leaf agents towards root. Message from a leaf agent is computed as follows.

Let the subnet of a leaf agent $B$ be over $(D, E, U)$, and its interface with the adjacent agent $C$ on hypertree be $S D \subset D$. Message $u t m_{0}(S D)$ that $B$ sends to $C$ is a MEU function that, for each local plan $\overline{s d}$ over $S D$, specifies

$$
u t m_{0}(\overline{s d})=\max _{\overline{r d}} e u(\overline{s d}, \overline{r d}),
$$

where $\overline{r d}$ is a local plan over $R D=D \backslash S D$. From Proposition 2, if $B$ applies PEUtilDec2 with $\beta=S D$, the return value meu $(\beta)$ satisfies $u t m_{0}(S D)=\operatorname{meu}(\beta)$. Since this computation is exponential on $\rho$, we seek to improve its efficiency below.

For each utility $u_{i}$ and its $\alpha_{i}$, we define $\beta_{i}=\alpha_{i} \cap S D$ and $\gamma_{i}=\alpha_{i} \backslash \beta_{i}$. That is, $\beta_{i}$ is public and $\gamma_{i} \subset R D$ is private relative to $C$. Utility variables can then be classified into four exhaustive and mutually exclusive cases:

1. $\gamma_{i}=\emptyset$. That is, $\alpha_{i}=\beta_{i}$ is entirely public.

2. $\gamma_{i} \neq \emptyset, \beta_{i} \neq \emptyset$, and $\gamma_{i} \cap \gamma_{j}=\emptyset$ for all $j \neq i$. That is, there exists no other utility variable $u_{j}$, such that $u_{i}$ and $u_{j}$ share a private decision ancestor.

3. $\gamma_{i} \neq \emptyset, \beta_{i} \neq \emptyset$, and $\gamma_{i} \cap \gamma_{j} \neq \emptyset$ for some $j \neq i$.

4. $\beta_{i}=\emptyset$. That is, $\alpha_{i}=\gamma_{i}$ is entirely private.

For example, suppose $S D=\left\{d_{2}, d_{3}\right\}$ in Fig. 2. Then $u_{2}$ is under case $1, u_{1}$ is under case $2, u_{3}$ is under case 3 , and $u_{4}$ is under case 4 .

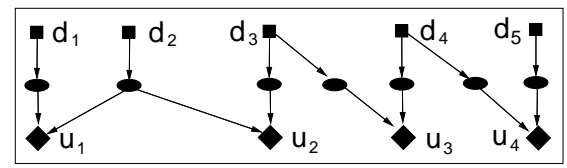

Fig. 2. Illustration of correlated cluster

[Case 1] For this case, $\overline{b_{i}}=\operatorname{proj}\left(\overline{s d}, \beta_{i}\right)=\operatorname{proj}\left(\overline{s d}, \alpha_{i}\right)$. We refer to the subnet segment that contains $u_{i}$ and all its ancestors (including $\pi_{i}$ and $\alpha_{i}$ ) as the subnet segment of $u_{i}$. For instance, subnet segment of $u_{2}$ in Fig. 2 includes nodes $d_{2}, d_{3}$, $u_{2}$, the two parent nodes of $u_{2}$ and links among them.

We observe that contribution of $u_{i}$ to Eqn. (11) can be evaluated using its subnet segment, independently of other $u_{j} \in U$, and the contribution is additive. That is, if $m e u(\overline{s d})=v$ is obtained using $B$ 's subnet, $m e u(\overline{s d})=v^{\prime}$ is obtained using 
$B$ 's subnet with node $u_{i}$ removed, and $e u\left(\overline{b_{i}}\right)=v^{\prime \prime}$ is obtained using the subnet segment of $u_{i}$, then $v=v^{\prime}+v^{\prime \prime}$. We therefore compute $e u\left(\alpha_{i}\right)$ using the subnet segment of $u_{i}$ according to Eqn. (3). As it involves full evaluation for each $\overline{b_{i}}$, the complexity is

$$
O\left(\sigma^{\left|\alpha_{i}\right|} m \kappa^{m}\right) .
$$

[Case 2] For this case, the contribution of $u_{i}$ to Eqn. (11) is also additive and obtainable from its subnet segment. Therefore, we can apply PEUtilDec1 to the subnet segment. Set the parameters of PEUtilDec1 to $D=\alpha_{i}, \beta=\beta_{i}$, and $\gamma=\gamma_{i}$. The return value $\operatorname{meu}\left(\beta_{i}\right)$ is the contribution of $u_{i}$ to Eqn. (11). Note that the return value peer $\left(\beta_{i}\right)$ will be used later and needs to be saved. Applying Proposition 1 to this case, the complexity to obtain $\operatorname{meu}\left(\beta_{i}\right)$ is then

$$
O\left(\theta \sigma^{\left|\alpha_{i}\right|} m \kappa^{m}\right) .
$$

[Cases 3 and 4] These utility variables can be grouped into correlated clusters (possibly overlapping): Each cluster is obtained by starting with one variable $u_{i}$ under case 3 . Note that whenever cases 1 and 2 do not cover all utility variables, there exist some under case 3 . Be definition, there exists $u_{j}$ such that $u_{i}$ and $u_{j}$ share a private decision ancestor. Note that $u_{j}$ may be under case 3 or case 4 . Add $u_{j}$ to the cluster, and continue until no such utility variable can be found. In Fig. 2, $u_{3}$ and $u_{4}$ form a correlated cluster. Formally, a correlated cluster can be defined as follows.

Definition 1 Let $S U=\left\{u_{1}, \ldots, u_{\eta^{\prime}}\right\} \subseteq U$ be a subset of utility variables. $S U$ is a correlated cluster if for each $u_{i}\left(i=2, \ldots, \eta^{\prime}\right)$, there exists $j<i$ with $\gamma_{j} \cap \gamma_{i} \neq \emptyset$, and no proper superset of $S U$ has such property.

For each correlated cluster with its utility variables indexed as $u_{1}, \ldots, u_{\eta^{\prime}}$, we apply PEUtilDec 2 to its subnet segment. Denote $\alpha^{\prime}=\cup_{i=1}^{\eta^{\prime}} \alpha_{i}, \beta^{\prime}=\cup_{i=1}^{\eta^{\prime}} \beta_{i}, \quad \gamma^{\prime}=$ $\cup_{i=1}^{\eta^{\prime}} \gamma_{i}$. Set parameters of PEUtilDec 2 to $D=\alpha^{\prime}, \beta=\beta^{\prime}$, and $\gamma=\gamma^{\prime}$. Return value $\operatorname{meu}\left(\beta^{\prime}\right)$ is contribution of the cluster to Eqn. (11). Return value $\operatorname{peer}\left(\beta^{\prime}\right)$ is needed later and is to be saved. Applying Proposition 2, complexity to obtain $\operatorname{meu}\left(\beta^{\prime}\right)$ is

$$
O\left(\theta \sigma^{\left|\alpha^{\prime}\right|} \eta^{\prime} m \kappa^{m}\right) .
$$

Let $S U_{1}, S U_{2}, \ldots$ be subsets of $U$, where $\cup_{i} S U_{i}=U$, and each $S U_{i}$ is either a singleton under case 1 , or a singleton under case 2 , or a correlated cluster from cases 3 or 4 . Let $\alpha_{i}^{\prime}, \beta_{i}^{\prime}$ and $\gamma_{i}^{\prime}$ denote the sets of decision ancestor variables for $S U_{i}$. Then Eqn. (11) can be computed as

$$
u t m_{0}(\overline{s d})=\left(\sum_{i} e u_{i}\left(\operatorname{proj}\left(\overline{s d}, \beta_{i}^{\prime}\right)\right)\right)+\sum_{j} \operatorname{meu}_{j}\left(\operatorname{proj}\left(\overline{s d}, \beta_{j}^{\prime}\right)\right),
$$

where each $e u_{i}()$ is the contribution from a $S U_{i}$ under case 1 , and each $m e u_{j}()$ is the contribution either from a $S U_{j}$ under case 2 or from a $S U_{j}$ under case 3 or 4. From Eqns. (12), (13) and (14), assuming cases 2, 3, 4 dominate the computation, the complexity to obtain $u t m_{0}(\overline{s d})$ is the following, where $\alpha^{*}=\max _{i} \alpha_{i}^{\prime}$,

$$
O\left(\theta \sigma^{\left|\alpha^{*}\right|} \eta m \kappa^{m}\right) .
$$


It is significantly more efficient than $O\left(\theta \sigma^{\rho} \eta m \kappa^{m}\right)$ (Proposition 2) as it would be if PEUtilDec 2 is directly applied to agent B's subnet. It is only exponential on cardinality of the largest cluster ancestor set, while the latter is exponential on $|D|$.

\section{$7 \quad$ Utility Message from Non-leaf}

Next, we consider non-leaf agents in the first round of message passing. Let $D$ be the set of decision variables of a non-leaf agent $B . B$ receives utility messages from child agents $A_{1}, \ldots, A_{k}$, over interfaces $S D_{1}, \ldots, S D_{k}$, respectively, and then computes and sends a utility message over interface $S D$ with parent agent $C$.

We denote message from $A_{j}$ by $u t m_{j}\left(S D_{j}\right)$, which specifies $u t m_{j}\left(\overline{s d_{j}}\right)$ for each local plan $\overline{s d_{j}}$ over $S D_{j}$. To count $u t m_{j}\left(S D_{j}\right)$ in computing message $u t m_{0}(S D)$ to $C$, we modify subnet as follows: For each decision variable $d_{i} \in S D_{j}$ with space $O p_{i}$, add a new child node $e_{i}$ with space $E f_{i}=O p_{i}$. Associate CPT $P\left(e_{i} \mid d_{i}\right)$ such that $P\left(e_{i} \mid d_{i}\right)=1$ whenever $e_{i}=d_{i}$ and $P\left(e_{i} \mid d_{i}\right)=0$ otherwise. Hence, $e_{i}$ is deterministically dependent on $d_{i}$. Denote the set of new child nodes added relative to $S D_{j}$ as $S E_{j}$. Add a new utility node $u t m_{j}$ with $S E_{j}$ as its parents, associate it with the function $u t m_{j}\left(S E_{j}\right)$, such that $u t m_{j}\left(S E_{j}\right)=u t m_{j}\left(S D_{j}\right)$, and assign it weight $w_{j}=1$.

After conversion for each $S D_{j}$, message $u t m_{0}(S D)$ to $C$ is computed by the method in Section 6 and Eqn. (15). The effect is that for each $\overline{s d}$ over $S D, u t m_{0}(\overline{s d})$ is the MEU based on all subnets on the hypertree rooted at the subnet of agent $B$.

For root agent, after conversion for each $S D_{j}$, it performs PEUtilDec2 with $\beta=\emptyset$ to get the optimal local plan $\overline{d^{*}}$. The first round of message passing ends. Operation by non-leaf agent $B$ is summarized in Algorithm 3. Its main property is established in Proposition 3 with proof omitted for space.

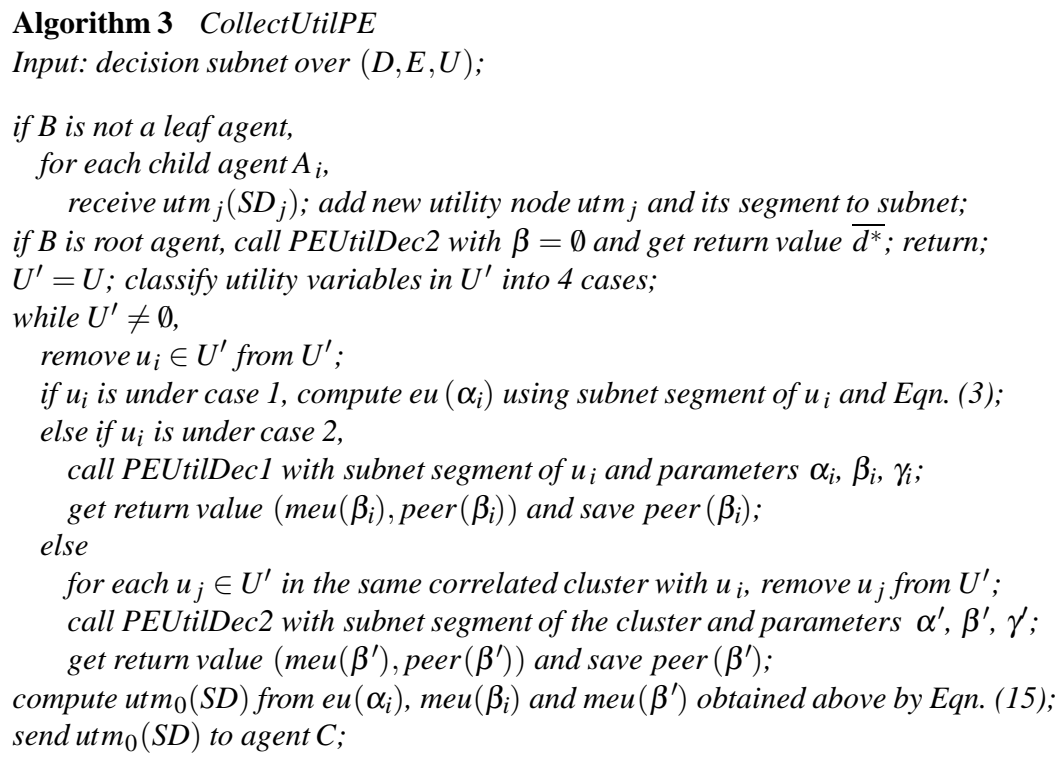


Proposition 3 For each non-root agent B, after completing CollectUtilPE, its message utm $m_{0}(S D)$ is the $M E U$ function with respect to joint plans over the union of subenvs on the sub-hypertree rooted at $B$.

\section{Decision Message Distribution}

The second round of message passing starts at root agent. It projects the optimal local plan to interface with each adjacent agent on hypertree, and sends the restricted plan to the agent. When a non-root agent $B$ receives the local plan $\overline{s d^{*}}$ over its interface $S D$ with the parent agent $C$, it uses the message to compute its optimal local plan. The computation is organized based on the partition of its utility variables into the four cases in Section 6.

If $u_{i}$ is under case 1 , then $\alpha_{i} \subset S D$, and the optimal local plan over $\alpha_{i}$ is

$$
\overline{s d_{i}^{*}}=\operatorname{proj}\left(\overline{s d^{*}}, \alpha_{i}\right) .
$$

If $u_{i}$ is under case $2, B$ obtains $\overline{b_{i}^{*}}=\operatorname{proj}\left(\overline{s d^{*}}, \beta_{i}\right)$, and retrieves $\overline{y_{i}^{*}}=\operatorname{peer}\left(\overline{b_{i}^{*}}\right)$ using the peer function saved during CollectUtilPE. The optimal local plan over $\alpha_{i}$ is

$$
\overline{s d_{i}^{*}}=\left(\overline{b_{i}^{*}}, \overline{y_{i}^{*}}\right) .
$$

If $u_{i}$ is under cases 3 or 4 , the optimal plan over its correlated cluster with decision ancestor sets $\alpha^{\prime}$ and $\beta^{\prime}$ is obtained in one operation (although we still index the result by $i$ ). $B$ obtains $\overline{b^{\prime *}}=\operatorname{proj}\left(\overline{s d^{*}}, \beta^{\prime}\right)$, and retrieves $\overline{y^{\prime *}}=\operatorname{peer}\left(\overline{b^{\prime *}}\right)$ using the peer function saved during CollectUtilPE. The optimal local plan over $\alpha^{\prime}$ is

$$
\overline{s d_{i}^{*}}=\left(\overline{b^{\prime *}}, \overline{y^{\prime *}}\right) \text {. }
$$

After the optimal local plan over each $\alpha_{i}$ (case 1 or 2 ) or $\alpha^{\prime}$ (case 3 or 4 ) is defined, the optimal local plan over $D$ (decision nodes in $B$ ) is the join

$$
\overline{d^{*}}=\left(\overline{s d_{1}^{*}}, \overline{s d_{2}^{*}}, \ldots\right) \text {. }
$$

The operation by $B$ is summarized in DistributePEPlan.

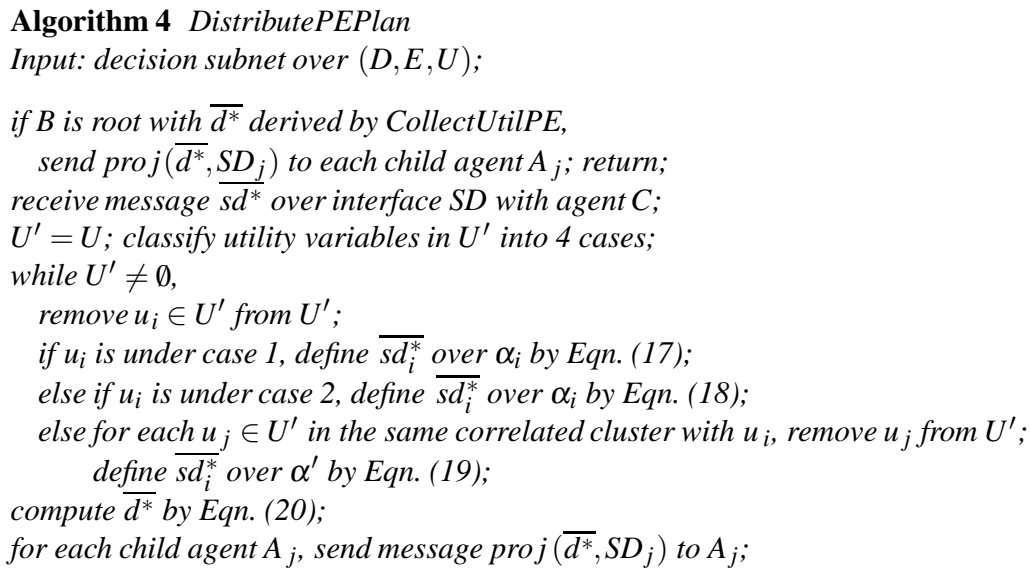


System coordinator executes DecisionPE, which combines above algorithms. Its optimality is established in Theorem 1, with proof omitted for space.

\begin{abstract}
Algorithm 5 DecisionPE
select an agent $C$ arbitrarily;

call CollectUtilPE in $C$;

call DistributePEPlan in C;
\end{abstract}

Theorem 1 After DecisionPE, the joint plan made from joining the local plan $\overline{d^{*}}$ at each agent is optimal.

As computation of root agent is dominated by the rest, from Eqn. (16), the total complexity of DecisionPE is $O\left(n \theta \sigma^{\left|\alpha^{*}\right|} \eta m \kappa^{m}\right)$, only exponential on size of the correlated cluster $\left|\alpha^{*}\right|$. This represents an exponential reduction from $\sigma^{\rho}$ to $\sigma^{\left|\alpha^{*}\right|}$ as well as a factor $\theta$ reduction, relative to the algorithm in [6].

\title{
9 Case Study in MAE
}

Three agent teams are formed to plan for horizon $h=1$. Two of them (referred to as $3 A 3 D$ and $3 A 5 D)$ each has 3 agents, and one $(5 A 5 D)$ has 5 agents. $3 A 3 D$ and $3 A 5 D$ are organized into hypertree $A-B-C$, and $5 A 5 D$ into $A-B-C-D-E$. Each decision has $\sigma=5$. Each subnet has between 1 and 3 public decision variables. Numbers of private decision variables per subnet in $3 A 3 D, 3 A 5 D, 5 A 5 D$ are $3,5,5$, respectively. Hence, the maximum numbers of decision variables per agent for the three teams are $6,8,8$, respectively. Maximum numbers of local plans per agent are $15625,390625,390625$, respectively. Numbers of joint plans for the teams are $6.1 \times 10^{9}, 9.5 \times 10^{13}, 1.4 \times 10^{25}$, respectively.

Three MAE envs are simulated of different reward distributions. Each team is placed at 6 distinct positions in each env, creating 18 distinct decision scenarios. For each scenario, each team is run using the method in [6] (denoted CDNCD) and that in this work (denoted CDNPE). For all teams and all decision scenarios, CDNPE runs obtained identical plans as CDNCD runs, confirming CDNPE optimality.

Table 1. Mean $\mu$ and standard deviation st $d$ for runtime (in seconds)

\begin{tabular}{c|cc|cc}
\hline \hline Team & \multicolumn{2}{|c|}{ CDNPE Time } & \multicolumn{2}{|c}{ CDNCD Time } \\
& $\mu$ & std & $\mu$ & std \\
\hline 3A3D & 3.41 & 0.05 & 13.9 & 0.92 \\
3A5D & 4.84 & 0.24 & 124 & 0.58 \\
5A5D & 4.78 & 0.25 & 127 & 0.63 \\
\hline \hline
\end{tabular}

Table 1 summarizes runtime per team and method. CDNPE runs significantly faster than CDNCD. For 3A3D (less expensive agents), CDNPE takes $25 \%$ of the time used by CDNCD. For 5A5D (more expensive), CDNPE takes $3.8 \%$ of the time. 


\section{Conclusion}

We extend multiagent decision algorithm in [6] and generalize partial evaluation for MAE in [9] to propose a new algorithm for length-2 CDNs under pivot probability assumption, reducing computation complexity exponentially compounded with a factor. In relation to MAIDs, ours is tightly coupled while MAIDs are loosely coupled [7]. In relation to DCOP methods such as DPOP, ours is decision theoretic while DPOP is not. Its generality rests on allowing pivot probability beyond a single value. Decision optimality is expected to degrade gracefully as pivot probability assumption is relaxed, and more experimental study is underway to confirm this.

\section{Acknowledgements}

Financial support from NSERC, Canada is acknowledged.

\section{References}

1. D.S. Bernstein, R. Givan, N. Immerman, and S. Zilberstein. The complexity of decentralized control of markov decision processes. Mathematics of Operations Research, 27(4):819-840, 2002.

2. F. Oliehoek, Matthijs Spaan, S. Whiteson, and N. Vlassis. Exploiting locality of interaction in factored Dec-POMDPs. In Proc. 7th Inter. Conf. on Autonomous Agents and Multiagent Systems, pages 517-524, 2008.

3. D.V. Pynadath and M. Tambe. The communicative multiagent team decision problem: Analyzing teamwork theories and models. J. Artificial Intelligence Research, 16:389-423, 2002.

4. M. Roth, R. Simmons, and M. Veloso. Exploiting factored representations for decentralized execution in multi-agent teams. In Proc. 6th Inter. Joint Conf. on Autonomous Agents and Multiagent Systems , pages 469-475, 2007.

5. Y. Xiang, J. Chen, and A. Deshmukh. A decision-theoretic graphical model for collaborative design on supply chains. In A.Y. Tawfik and S.D. Goodwin, editors, Advances in Artificial Intelligence, LNAI 3060, pages 355-369. Springer, 2004.

6. Y. Xiang, J. Chen, and W.S. Havens. Optimal design in collaborative design network. In Proc. 4th Inter. Joint Conf. on Autonomous Agents and Multiagent Systems (AAMAS'05), pages 241-248, 2005.

7. Y. Xiang and F. Hanshar. Comparison of tightly and loosely coupled decision paradigms in multiagent expedition. International Journal of Approximate Reasoning, 51:600-613, 2010.

8. Y. Xiang and F. Hanshar. Multiagent expedition with graphical models. Inter. J. Uncertainty, Fuzziness and Knowledge-Based Systems , 19(6):939976, 2011.

9. Y. Xiang and F. Hanshar. Partial evaluation for planning in multiagent expedition. In C. Butz and P. Lingras, editors, Advances in Artificial Intelligence, LNAI 6657, pages 420-432. Springer, 2011.

10. W. Zhang and Q. Ji. A factorization approach to evaluating simultaneous influence diagrams. IEEE SMC-A, 36(4):746-757, 2006. 